\title{
Efecto de la restricción calórica sobre la composición corporal y la capacidad de salto en jugadoras de fútbol
}

\author{
Effect of Calorie Restriction on Body Composition and Jump Capacity in the \\ Female Soccer Players \\ Efeito da restrição calórica na composição corporal e capacidade de salto nos jogadores \\ de futebol feminino
}

\author{
Gloria I. García-Morales', Miguel A Niño-Rey', Ildefonso Alvear-Ordenes²
}

Recibido: 16 de julio de 2019. Aceptado para publicación: 5 de agosto de 2019 https://doi.org/10.35454/rncm.v2n2.003

\section{Resumen}

Introducción: mejorar la composición corporal en jugadoras de fútbol trae beneficios para el desarrollo de cualidades físicas específicas.

Objetivo: valorar el efecto de una restricción calórica leve sobre la composición corporal y la capacidad de salto de jugadoras de fútbol.

Métodos: se seleccionaron 18 jugadoras de la Selección Nacional de Colombia. Se subdividieron utilizando como criterio la suma de 6 pliegues ( $\Sigma 6 \mathrm{skf})$, en un grupo con exceso de tejido adiposo ( $\Sigma 6 \mathrm{skf}>70$ $\mathrm{mm}$ ) o grupo experimental, y un grupo con tejido adiposo normal ( $\Sigma 6 \mathrm{skf}$ entre 40 - $70 \mathrm{~mm}$ ) o grupo control. Ambos grupos siguieron el mismo protocolo de entrenamiento (16 meses). La dieta normal establecida para el grupo control $(2.650 \mathrm{kcal} /$ día) se redujo en el grupo experimental (20\% de restricción calórica, $2.135 \mathrm{kcal} /$ día). Se midió la composición corporal por antropometría (5 componentes) y capacidad de salto con 4 tipos diferentes de salto (salto sin impulso, salto contra-movimiento, salto Abalakov, y salto máximo).

\section{Summary}

Introduction: Improving the body composition of soccer players is beneficial for the development of specific physical qualities.

Objective: To assess the effect of a mild calorie restriction on body composition and jump capacity of the female soccer players.

Methods: Eighteen players from the Colombian national team were selected. They were subdivided using as criteria the sum of 6 folds, in a group with excess adipose tissue (sum of six folds $>70 \mathrm{~mm}$ ) or experimental group and a group with normal adipose tissue (sum of six folds between $40-70 \mathrm{~mm}$ ) or control group. Both groups followed the same training protocol (16 months). The normal diet established for the control group (2650 $\mathrm{kcal} /$ day) was reduced in the experimental group (20\% caloric restriction, $2135 \mathrm{kcal} /$ day). Body composition was measured by anthropometry (5 components) and jump capacity with 4 different types of jump (squat jump, counter movement jump, Abalakov jump, maximum jump).

\section{Resumo}

Introdução: a melhoria da composição corporal de jogadores de futebol feminino é benéfica para o desenvolvimento de qualidades físicas específicas.

Objetivo: avaliar o efeito de uma restrição calórica leve sobre a composição corporal e capacidade de salto de jogadores de futebol feminino.

Método: 18 jogadores da seleção colombiana de futebol feminino foram selecionados. Elas foram subdivididas usando como critério a soma de 6 dobras ( $\Sigma 6 \mathrm{skf})$, em um grupo com excesso de tecido adiposo ( $\Sigma 6 \mathrm{skf}>70 \mathrm{~mm}$ ) ou grupo experimental, e um grupo com tecido adiposo normal ( $\Sigma 6 \mathrm{skf}$ entre 40 - $70 \mathrm{~mm}$ ) ou grupo de controle.

Ambos os grupos seguiram o mesmo protocolo de treinos (16 meses). A dieta normal estabelecida para o grupo controle (2.650 kcal / dia) foi reduzida no grupo experimental (20\% de restrição calórica, 2.135 $\mathrm{kcal} / \mathrm{dia})$. A composição corporal foi medida por antropometria (5 componentes) e a capacidade de salto, com 4 tipos diferentes de salto (salto sem impulso, salto contra
Facultad de Medicina y posgrado de Medicina del Deporte, Universidad El Bosque, Bogotá, D.C., Colombia.

Instituto de Biomedicina (IBIOMED), Universidad de León, León, España
Correspondence: *Ildefonso Alvear-Ordenes ialvor@unileon.es 
Resultados: el grupo experimental mostró cambios significativos; disminución de masa adiposa (kg y \%) y suma de seis pliegues; y aumento de masa muscular (kg y \%) e índice músculo/ óseo. Además, el entrenamiento unido a una restricción calórica leve mostró un potente efecto sobre el peso corporal, el índice de masa corporal, sumatoria de seis pliegues y porcentaje de masa muscular.

Conclusiones: los datos indican que a menor masa adiposa, mayor capacidad de salto y mientras más altas y delgadas sean las jugadoras, saltarán más. Una mayor masa muscular no parece ser requerida para tener mayor capacidad de salto. Aunque la restricción calórica produce un potente efecto en la composición corporal, no lo hace sobre la capacidad de salto.

Palabras clave: restricción energética, dieta, antropometría, potencia de salto, fútbol femenino.
Results: The experimental group showed significant changes; decreased fat mass ( $\mathrm{kg}$ and \%) and sum of six folds; and increased muscle mass ( $\mathrm{kg}$ and \%) and muscle/skeletal index. In addition, training in conjunction with a mild caloric restriction showed a potent effect on body mass, body mass index, sum of six folds, adipose mass ( $\mathrm{kg}$ and $\%$ ) and muscle mass percentage.

Conclusions: The data indicates that the lower the adipose mass, the higher the jump capacity and the taller and thinner the players are, the more they will jump. A larger muscle mass does not appear to be required to have a higher jump capacity. Although calorie restriction has a powerful effect on body composition, it does not influence jump capacity.

Keywords: Energy restriction; Diet; Anthropometry; Jumping power; Women's soccer. movimento, salto Abalakov e salto máximo).

Resultados: o grupo experimental apresentou alterações significativas; diminuição da massa adiposa ( $k$ e \%) e soma de seis dobras; e aumento da massa muscular (kg e \%) e índice músculo / osso. Além disso, o treino associado a uma restrição calórica leve, mostrou um efeito potente sobre o peso corporal, índice de massa corporal, soma de seis dobras e percentagem de massa muscular.

Conclusões: os dados indicam, que a menor massa adiposa maior capacidade de salto e quanto maior e mais magras as jogadoras, mais elas saltarão. Maior massa muscular não parece ser necessária para ter maior capacidade de salto. Embora a restrição calórica produza um potente efeito na composição corporal, ela não afeta a capacidade de salto.

Palavras-chave: restrição energética, dieta, antropometria, força de salto, futebol feminino.

\section{INTRODUCCIÓN}

El fútbol femenino es cada día más popular entre las mujeres y el desarrollo de cualidades físicas específicas se beneficia en forma notable cuando existe una adecuada composición corporal ${ }^{(1)}$. Además, la restricción calórica acompañada de ejercicio de mediana intensidad causa mejoras importantes en la composición corporal ${ }^{(2)}$.

Muchos parámetros de la composición corporal pueden ser utilizados en el fútbol competitivo, así como en otros deportes de élite, para el control deportivo ${ }^{(1,3,4)}$. En la mujer futbolista la suma de seis pliegues ( $\Sigma 6 \mathrm{skf:}$ tríceps + subescapular + supra espinal + abdominal + muslo frontal + pantorrilla) entre 45 y $65 \mathrm{~mm}$ de tejido adiposo, se relaciona con el mantenimiento de la salud, de las funciones reproductivas y del sistema inmune, así como a una mayor velocidad, agilidad, resistencia y potencia de salto ${ }^{(1,4,5)}$.

Una ingesta adecuada de energía se asocia a un rendimiento deportivo óptimo ${ }^{(6)}$. El gasto energético por sesión de entrenamiento fluctúa entre $600-950 \mathrm{kcal}^{(7)}$ con una intensidad del ejercicio entre $70 \%-75 \%$ del $\dot{\mathrm{V}}{ }_{2 \max }{ }^{(7)}$. Se calcula que las necesidades de energía pueden estar entre 40 y $50 \mathrm{kcal} / \mathrm{Kg} /$ día, es decir, 2.200 - $2.800 \mathrm{kcal}$ / día para una jugadora de entre 56 y $62 \mathrm{~kg}$ de peso corporal. Por lo anterior, las deportistas deben aprender a seguir una dieta que promueva una buena salud, evite las deficiencias nutricionales, cubra las necesidades de nutrientes esenciales para la mujer (hierro, calcio y ácido fólico), además de las demandas de energía del entrenamiento y de otras actividades diarias ${ }^{(8)}$.

En el fútbol femenino, muchas jugadoras no logran mantener un equilibrio energético que favorezca su composición corporal, algunas restringen su ingesta de alimentos para alcanzar el peso deseado, afectando su rendimiento y salud. Otras, por el contrario, se exceden en el aporte de calorías, aumentando las reservas de tejido adiposo ${ }^{(9)}$. Por lo tanto, en un deporte donde la resistencia y la potencia aeróbica determinan el rendimiento físico un exceso de peso en tejido adiposo podrá afectar su desempeño deportivo. Además, las prácticas inseguras o inadecuadas utilizadas para el control del peso corporal, sin supervisión de un profesional, ponen en riesgo la salud del deportista ${ }^{(5)}$.

La dieta de las jugadoras de futbol debe ser mixta, alta en carbohidratos ( $\mathrm{CHO}$ ), con un promedio de $8 \mathrm{~g}$ de $\mathrm{CHO} / \mathrm{kg} /$ día. El aporte de proteína debe ser más alto que el recomendado para la población de mujeres adultas sanas, $\left(1,4\right.$ a $\left.1,7 \mathrm{~g} \cdot \mathrm{kg}^{-1} \cdot \mathrm{dia}^{-1}\right)$, pre y postejercicio, para evitar el catabolismo proteico durante el ejercicio de alta intensidad, favorecer el mantenimiento de la masa muscular y alcanzar una adecuada recuperación 
postentrenamiento y competencia ${ }^{(10,11)}$, así como para una mayor utilización de los ácidos grasos durante el ejercicio $^{(12)}$.

La velocidad y la potencia son características físicas de gran importancia en el fútbol; el desarrollo de la fuerza explosiva de miembros inferiores es indispensable para alcanzar la capacidad de salto deseada ${ }^{(13)}$. En el deporte la capacidad de salto (CS) es evaluada normalmente a través del "test de Bosco", utilizando los diferentes tipos de salto como el salto sin impulso (SJ), salto contra - movimiento (CMJ), salto Abalakov $(\mathrm{ABLKJ})$ y salto máximo $(\mathrm{MJ})^{(2,14)}$.

Una restricción calórica leve, junto con un programa de entrenamiento específico para el fútbol femenino, podría ajustar la composición corporal de las jugadoras, haciéndolas más atléticas, con menor cantidad de masa adiposa y mayor masa muscular, además de favorecer una de las capacidades físicas más importantes en el futbol, como es la capacidad de salto. La aplicación de un plan alimentario completo y balanceado, suficiente en carbohidratos, modificado en azúcares simples añadidos y bajo en grasas saturadas visibles en la dieta, puede contribuir a este proceso.

El objetivo de este estudio fue evaluar el efecto de una restricción calórica leve, equivalente a una reducción de $20 \%$ de las calorías recomendadas, sobre la composición corporal y la capacidad de salto en un grupo de jugadoras de fútbol profesional con exceso de tejido adiposo.

\section{MÉTODOS}

\section{Muestra}

De las 25 jugadoras de la Selección Nacional de Fútbol de Colombia, se seleccionaron 18 de ellas para formar una muestra longitudinalmente activa, homogénea, conveniente y representativa de la élite del fútbol competitivo femenino del país. Criterios de inclusión: a. haber participado en $80 \%$ de las convocatorias (6 como mínimo); b. haber participado en un mínimo de 3 competiciones internacionales; c. tener mínimo 6 años de experiencia en el plano competitivo; $y$, d. haber tenido mínimo 10 horas de entrenamiento de fútbol/ semana en los últimos dos años. Además, las jugadoras no podían consumir suplementos dietéticos, ni como reemplazo de su alimentación, ni para reducir el apetito o perder masa corporal. Todas las jugadoras pasaron un reconocimiento médico que las autorizó para la competición de élite, se familiarizaron con el protocolo y con los posibles riesgos potenciales y dieron su consentimiento informado por escrito para participar en el estudio. El protocolo fue aprobado por la Unidad de Posgrado (Máster), de la Universidad de León, exigiendo todos los criterios de la Declaración de Helsinki de 1975, revisada en 2008.

La muestra se dividió en dos grupos según los criterios de inclusión. El grupo control compuesto por 9 jugadoras con una masa corporal (MC) adecuada para su talla (según IMC y posición de juego) y una ¿6skf entre 45 - $70 \mathrm{~mm}$. El grupo experimental con 9 jugadoras con exceso de tejido adiposo o sobrepeso, con elevado IMC (por encima de $24,5 \mathrm{~kg} / \mathrm{m}^{2}$ ) y que mostraban una $\Sigma 6 \mathrm{skf}$ mayor a $65 \mathrm{~mm}$ para jugadoras de campo y de $70 \mathrm{~mm}$ para las porteras. El límite de la $\Sigma 6$ skf entre jugadoras de campo y porteras se basó en los criterios y características de los deportistas de élite $(\text { Tabla } 1)^{(1,4)}$.

\section{Antropometría}

Al inicio y al finalizar la intervención de la dieta se midió, además de las medidas básicas de peso corporal $(\mathrm{kg})$ talla y talla sentado $(\mathrm{cm}), 6$ diámetros (biacro-

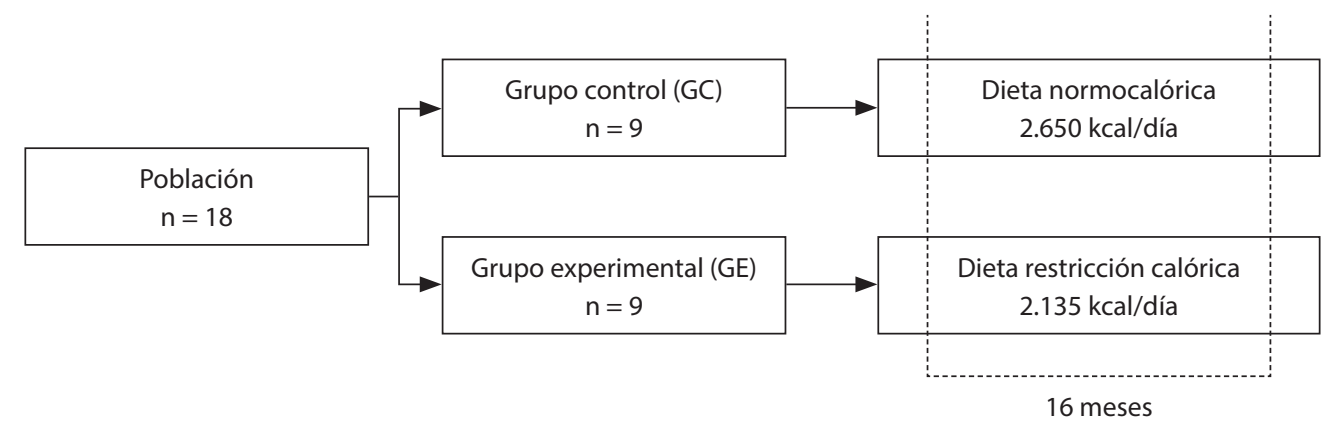

Figura 1. Diseño experimental del estudio. La dieta del grupo control fue calculada con una restricción de $20 \%$. 
mial, tórax trasverso, tórax antero-posterior, bi-iliocrestal, húmero y fémur), 10 perímetros (cabeza, brazo relajado, brazo flexionado y tensionado, antebrazo máximo, muñeca, tórax meso-esternal, cintura mínima, muslo máximo, muslo medio, pantorrilla) y 6 pliegues cutáneos (tríceps, subescapular, supra espinal, abdominal, muslo medio y pantorrilla $)^{(15)}$. Las 25 medidas utilizadas para el análisis de la composición corporal por el método de cinco componentes anatómicos de masa (piel, masa adiposa (MA), masa muscular (MM), tejido óseo y tejido residual) conforma un método validado en salud pública ${ }^{(16)}$. Midiéndose también el índice músculo/ óseo o IM-O (IM-O = MM, kg/ Masa ósea, $\mathrm{kg})^{(3)}$. Las evaluaciones antropométricas se realizaron siempre por la mañana, antes de la sesión de entrenamiento o en día de descanso y por un antropometrista certificado (ISAK, nivel III), con un error técnico de medida de $1,17 \%$.

Tabla 1. Características de la muestra $(n=18)$. Los datos se muestran como valores medios y desviaciones estándar ( \pm SD)

\begin{tabular}{|l|c|}
\hline \multicolumn{1}{|c|}{ Variables } & Media y SD \\
\hline Edad (años) & $21,4 \pm 3,1$ \\
\hline Talla (cm) & $164,4 \pm 4,9$ \\
\hline Masa corporal $(\mathrm{kg})$ & $59,8 \pm 7,4$ \\
\hline Índice de masa corporal $\left(\mathrm{kg} / \mathrm{m}^{2}\right)$ & $22,1 \pm 2,5$ \\
\hline Suma de 6 pliegues $(\mathrm{mm})$ & $79,8 \pm 37,6$ \\
\hline Masa adiposa (\%) & $28,5 \pm 6,0$ \\
\hline Masa muscular (\%) & $45,5 \pm 4,0$ \\
\hline Índice músculo/ óseo $(\mathrm{kg})$ & $4,6 \pm 0,29$ \\
\hline Endomorfa & $3,5 \pm 1,6$ \\
\hline Mesomorfa & $3,6 \pm 0,9$ \\
\hline Ectomorfa & $2,3 \pm 1,2$ \\
\hline
\end{tabular}

\section{Procedimiento y dieta}

Antes de iniciar los 16 meses de intervención de la dieta, se evaluó la ingesta mediante anamnesis alimentaria de 3 días, individualmente y en diferentes situaciones de entrenamiento y alimentación. Se evaluó también la frecuencia de consumo de alimentos y los hábitos y conductas alimentarias, para orientar la educación nutricional durante la intervención ${ }^{(5,17,18)}$. El gasto energético total se calculó con la fórmula de Harris y Benedict para gasto energético basal ${ }^{(19)}$, se sumó el efecto térmico de los alimentos (+10\%), las actividades diferentes al entrenamiento $(+25 \%)$ y el gasto energético por entrenamiento ${ }^{(11)}$. Este último se calculó a partir del compendio de actividades físicas ${ }^{(20)}$, calculando el promedio de las actividades de 7 días ${ }^{(11)}$. Con el cálculo del gasto energético total diario se definió la fórmula dietaria recomendada para el grupo de jugadoras que conformaron el grupo control (Tabla 2) y la prescrita para el grupo experimental (Tabla 3), tanto para la ingesta nutricional durante las concentraciones deportivas como cuando permanecían en sus casas. El plan alimentario fue evaluado y controlado diariamente.

La dieta que recibió el grupo control (2.650 kcal.día ${ }^{-1}$; $15 \%$ proteína, $27 \%$ grasa y $58 \%$ carbohidratos) se caracterizó por ser normocalórica, hiperproteica leve, normograsa, hiperglúcida leve, baja en azúcares simples añadidos y en grasas saturadas, alta en agua, fibra y antioxidantes (Tabla 2). La dieta del grupo experimental fue una dieta de restricción calórica leve $\left(2.135 \mathrm{kcal} \cdot \mathrm{día}^{-1} ; 20 \%\right.$ proteínas, $25 \%$ grasa y $55 \%$ carbohidratos) con $20 \%$ menos del valor calórico recomendado ${ }^{(17)}$, se diferenció por ser normoglúcida, sin azúcares simples añadidos, muy baja en grasas saturadas y fuentes de colesterol, pero igualmente hiperproteica leve (Tabla 3).

\section{Test de salto}

La capacidad de salto (CS) se evaluó mediante la prueba de Bosco, con cuatro tipos de salto: SJ, CMJ, ABLKJ y $\mathrm{MJ}^{(13,17)}$.

\section{Entrenamiento}

Todas las jugadoras siguieron el mismo plan de entrenamiento específico de fútbol, similar en técnica, táctica y fuerza, con 9 - 10 sesiones por semana (aeróbico: 2 - 3 sesiones; fuerza con máquinas: 2 - 3 sesiones; y resto: sesiones técnicas).

\section{Análisis estadístico}

Los datos se presentan como media y desviación estándar ( \pm SD). Para la normalidad de las variables se utilizaron los Standard Skewness y Kurtosis. Las diferencias entre variables se establecieron mediante análisis de la varianza y la prueba de Newman-Keuls. La relación entre variables se realizó mediante el coeficiente de correlación ( $r$ ) de Pearson y, para las variables que no mostraron normalidad, se utilizó la $r$ de Sperman. El efecto del tratamiento se analizó por la diferencia entre 
Tabla 2. Fórmula dietaria recomendada para el grupo control o CG $(n=9)$. Ajustada a $2.650 \mathrm{Kcal} /$ día, para un sujeto con masa corporal (BM) de $56 \mathrm{Kg}$.

\begin{tabular}{|c|c|c|c|c|}
\hline Nutrientes & Cantidad (g) & Energía (kcal) & Proporción (\%) & Por kg de $M C\left(\mathbf{g} \cdot \mathbf{k g}^{-1}\right)$ \\
\hline Proteínas & 99,4 & 398 & 15 & 1,77 \\
\hline Grasas & 79,5 & 716 & 27 & 1,42 \\
\hline Carbohidratos & 384 & 1.537 & 58 & 6,86 \\
\hline Total & 562,9 & 2.651 & 100 & $10,05 *$ \\
\hline Agua & $\begin{array}{l}4.000 \mathrm{cc} \cdot \mathrm{dí}^{-1} \text { (líquido de la dieta + líquido en } \\
\text { entrenamiento) }\end{array}$ & & con $1,5 \mathrm{cc} \cdot \mathrm{kcal}^{-1} \cdot \mathrm{dí}^{-1}$ & \\
\hline
\end{tabular}

MC, masa corporal; * se estimó un consumo de $47,3 \mathrm{kcal} \cdot \mathrm{kg}^{-1}$.

Tabla 3. Fórmula dietaría prescrita para el grupo de jugadoras con exceso de tejido adiposo o CG ( $n=9$ ). Ajustada a 2.135 $\mathrm{Kcal} / \mathrm{día}$, para un sujeto con masa corporal (BM) de $61 \mathrm{Kg}$.

\begin{tabular}{|c|c|c|c|c|}
\hline Nutrientes & Cantidad (g) & Energía (kcal) & Proporción (\%) & Por kg de $\mathrm{MC}\left(\mathbf{g} \cdot \mathbf{k g}^{-1}\right)$ \\
\hline Proteínas & 107 & 427 & 20 & 1,75 \\
\hline Grasas & 59 & 534 & 25 & 0,97 \\
\hline Carbohidratos & 293 & 1.174 & 55 & 4,80 \\
\hline Total & 459 & 2.135 & 100 & $7,52 *$ \\
\hline Agua & \multicolumn{2}{|c|}{4.200 cc $\cdot$ día $^{-1}$ (líquido de la dieta + líquidos en entrenamiento) } & \multicolumn{2}{|c|}{$2 \mathrm{cc} \cdot \mathrm{kcal}^{-1} \cdot \mathrm{día}^{-1}$} \\
\hline
\end{tabular}

MC, masa corporal; * para un consumo estimado de $35 \mathrm{kcal} \cdot \mathrm{kg}^{-1}$.

los grupos control y experimental, de los cambios de los valores de cada variable presentados entre el inicio y la finalización de la intervención. El valor mínimo de significancia se estableció en un valor de $p<0,05$.

\section{RESULTADOS}

La edad de la población $(\mathrm{n}=18)$ fue de 21,4 $\pm 3,09$ años, con una talla de $164,4 \pm 4,91 \mathrm{~cm}$, una masa corporal de 59,8 $\pm 7,4 \mathrm{~kg}$, un índice de masa corporal (IMC) de $22,1 \pm 2,5 \mathrm{~kg} / \mathrm{m}^{2} \mathrm{y}$ un porcentaje de masa adiposa de $28,5 \pm 6,0 \%$. En la Tabla 1 se muestran las características de la muestra estudiada $(\mathrm{n}=18)$.

Después de la intervención de 16 meses con una dieta con restricción calórica leve, el grupo de estudio mostró cambios significativos $(\mathrm{p}<0,05)$ en parámetros antropométricos como $\Sigma 6 \mathrm{skf}$, Masa adiposa (en $\mathrm{kg}$ y $\%), \mathrm{MM}$ (en kg y \%) e índice musculo-óseo (IM-O), así como para la capacidad de salto (CS) medida con la mayoría de los tipos de salto (SJ, ABLKJ y MJ). El grupo control solo mostró cambios significativos $(\mathrm{p}<$
$0,05)$ en la MM ( kg) y en la capacidad de salto medida por la técnica de MJ.

En cuanto a la comparación entre los dos grupos (efecto de la restricción calórica leve: que corresponde a la suma de las diferencias encontradas en ambos grupos, para cada una de las variables estudiadas) se muestran cambios significativos para la masa corporal (MC), índice de masa corporal (IMC), suma de seis pliegue ( $56 \mathrm{skf}$ ), masa adiposa (MA) (en kg y \%), masa muscular (MM) (en \%), así como en el componente ectomorfo. En la Tabla 4 se muestran los resultados de cada grupo antes y después de la intervención, así como la comparación de los cambios observados en los dos grupos.

Los datos del análisis de correlación entre la capacidad de salto y las diferentes variables antropométricas estudiadas se muestran en la Tabla 5. En ella se observan los resultados entre los cuatro tipos de salto (SJ, $\mathrm{CMJ}, \mathrm{ABLKJ}, \mathrm{MJ})$ con las variables antropométricas. Se observan fuertes correlaciones $(-0,7<\mathrm{r}>0,7)$ para CMJ y ABLKJ con el IMC y el estado ectomorfo; para CMJ, ABLKJ y MJ para $\Sigma 6$ skf; y para SJ, CMJ y ABLKJ 
con la condición mesomorfa; todos ellos con un valor de $p<0,001$. Todas estas correlaciones fueron negativas, excepto las de la condición ectomorfa.

\section{DISCUSIÓN}

Nuestro estudio permite demostrar que una restricción calórica de $20 \%$, es decir, un déficit aproximado de 500 $\mathrm{kcal} \cdot \mathrm{dí}^{-1}$ fue suficiente para provocar cambios positivos en la composición corporal de jugadoras de futbol principalmente en la reducción de masa adiposa, mejora del porcentaje de masa muscular y de la relación músculo/ ósea ${ }^{(1)}$. Además, al ajustar la composición corporal de las jugadoras con exceso de tejido adiposo y mejorar el porcentaje de masa muscular, el perfil antropométrico de todas las jugadoras de la muestra fue más homogéneo y más cercano a las jugadoras consideradas de élite. Se confirma con ello el efecto significativo de la restricción calórica sobre la MC y sobre el IMC del grupo experimental, cambios que indicarían que una dieta con restricción calórica leve provoca un efecto determinante en la reducción de estas variables.

La talla y masa corporal del total de la muestra estudiada son similares a las de las selecciones de Brasil, México y Japón (campeona del mundo 2012) (21) (Tabla 1). Se diferencian un poco de otras rivales directas de competición como por ejemplo EE. UU, Canadá, Suiza y Alemania, que poseen un biotipo más fuerte ${ }^{(21)}$. La $\Sigma 6$ skf del grupo control mostró valores cercanos a la élite internacional $(50-70 \mathrm{~mm})$, mientras que las jugadoras del grupo experimental presentaban valores similares $(93-120 \mathrm{~mm}$ ) a jugadoras de menor nivel de rendimiento ${ }^{(22)}$. Por todo ello, los criterios utilizados en este estudio para dividir a las jugadoras en grupo control y experimental continúan siendo válido ${ }^{(1,4)}$.

La restricción calórica leve tuvo efectos positivos sobre la $\Sigma 6$ skf en el grupo de estudio $(\sim 30 \mathrm{~mm})$, la cual representa la reserva energética en diferentes partes del organismo y tiene una elevada correlación con la masa adiposa total ${ }^{(23)}$. En ambos grupos aumentó la MM, demostrando que la dieta utilizada preservó y mejoró este componente lo que ha sido demostrado en otros estudios $^{(12,24)}$.

Ambos grupos realizaron el mismo entrenamiento de 16 meses, aumentando en la misma proporción la MM $(\mathrm{kg})$, una situación que ha sido observada tanto en sujetos entrenados como no entrenados ${ }^{(27,29)}$. En los estudios de Kreider et al. y Milanovic et al. ${ }^{(28,29)}$, donde se estudiaron cambios en la composición corporal en personas no entrenadas y entrenadas y en deportistas, jugadoras de futbol juveniles, también se observó un aumento de la fuerza máxima y de la resistencia muscular, sin necesidad de una suplementación de proteínas adicional o cambios significativos de la dieta consumida ${ }^{(24-26)}$.

El índice músculo-óseo, considerado un indicador de rendimiento biomecánico mostró un significativo aumento en el grupo estudio ( $>7$ veces), provocado por un desarrollo importante de la MM (Tabla 4), con valores por encima de lo esperado (de 3,8 a 4,6) para deportistas mujeres ${ }^{(3)}$. Este índice que puede ser muy útil en la selección de talentos deportivos o determinar posiciones de juego (los delanteros centrales, con mayor potencia biomecánica, poseen un índice músculo-óseo de 4,77 y los mediocampistas muestran valores entre 4,44-4,47), por lo que es interesante el desarrollo de estudios que relacionen este índice con pruebas de potencia en campo, capacidad de salto y de velocidad, que pueden verse influidos por las diferencias de $\mathrm{MA}^{(3)}$.

En el grupo experimental se observaron importantes cambios de la MA (\%) y solo moderados para la MM (\%), esta última aumentó a un valor cercano a $48 \%$, valor superior al encontrado en otros estudios con jugadoras de fútbol suramericano ${ }^{(1)}$, aunque esperables en el fútbol femenino ${ }^{(2,19)}$ y en la mujer atleta ${ }^{(3,27)}$. La restricción calórica leve provocó un efecto positivo en la reducción de masa adiposa (\%).

En ninguno de los grupos los cambios en somatotipo fueron significativos. Sin embargo, se observó un efecto moderado de la restricción calórica sobre el componente ectomorfo, producido por el alejamiento en la modificación de este componente (grupo control: - 0,28 y grupo estudio: 0,44$)$. El grupo control mantuvo la condición meso ectomorfa, situación esperada en este tipo de deportistas ${ }^{(28)}$. Esto también se ha observado en los equipos de élite (EEUU, Canadá y Japón) finalistas del campeonato mundial FIFA $2012^{(18)}$. Aunque un somatotipo meso-ectomorfo parece favorecer el rendimiento físico, esta característica varía mucho entre las mejores jugadoras del mundo o dentro de un mismo equipo ${ }^{(29)}$. Quizá por ello en la selección de talentos se pone más atención a la habilidad y técnica específica que a las cualidades físicas ${ }^{(6)}$. El grupo estudio, en cambio, modificó levemente su somatotipo meso endomórfico, al disminuir la condición endomorfa y aumentar la ectomorfa (Tabla 4); una característica encontrada en jugadoras de fútbol no profesionales, que trabajan con volúmenes y cargas menores de entrenamiento que las jugadoras de este estudio ${ }^{(1,4,30)}$. 
Tabla 4. Diferencias observadas entre el CG y el EG, entre las evaluaciones iniciales y finales aplicadas a cada grupo durante el estudio. Se comparan las diferencias entre el inicio y la finalización de la intervención, para ambos grupos valorando el efecto del tratamiento de la restricción calórica sobre la muestra experimental de jugadoras con exceso de tejido adiposo. Los datos se muestran como valores medios y desviaciones estándar ( \pm SD). Las diferencias significativas se señalan a partir de un valor de $p<0,05$.

\begin{tabular}{|c|c|c|c|c|c|c|c|c|c|c|}
\hline \multirow[t]{2}{*}{ Variable } & \multicolumn{4}{|c|}{ Jugadoras - Grupo Control (GC) } & \multicolumn{4}{|c|}{ Jugadoras - con Exceso de Grasa (GE) } & \multicolumn{2}{|c|}{ Efecto } \\
\hline & Inicial & Final & Diferencia & $p$ & Inicial & Final & Diferencia & $p$ & Diferencia & $p$ \\
\hline Talla (cm) & $164,7 \pm 5,9$ & $165,1 \pm 5,6$ & $0,42 \pm 0,58$ & 0,061 & $164,1 \pm 4,0$ & $164,2 \pm 3,8$ & $0,13 \pm 0,33$ & 0,300 & $0,29 \pm 0,27$ & 0,331 \\
\hline $\begin{array}{l}\text { Masa } \\
\text { corporal (kg) }\end{array}$ & $54,8 \pm 4,9$ & $56,7 \pm 4,5$ & $1,86 \pm 3,68$ & 0,168 & $64,7 \pm 6,2$ & $62,0 \pm 7,2$ & $-2,7 \pm 3,7$ & 0,060 & $4,56 \pm 3,0$ & $<0,001$ \\
\hline BMI $\left(\mathrm{kg} / \mathrm{m}^{2}\right)$ & $20,2 \pm 1,7$ & $20,8 \pm 1,6$ & $0,59 \pm 1,39$ & 0,224 & $23,9 \pm 1,4$ & $22,9 \pm 1,8$ & $-1,06 \pm 1,4$ & 0,057 & $1,65 \pm 1,2$ & $<0,01$ \\
\hline $\begin{array}{l}\text { Talla sentada } \\
(\mathrm{cm})\end{array}$ & $84,9 \pm 2,6$ & $85,0 \pm 2,4$ & $0,12 \pm 0,37$ & 0,351 & $84,2 \pm 1,7$ & $84,6 \pm 1,7$ & $0,38 \pm 0,62$ & 0,105 & $0,26 \pm 0,22$ & 0,286 \\
\hline $\begin{array}{l}\text { Longitud de } \\
\text { piernas }(\mathrm{cm})\end{array}$ & $79,8 \pm 4,3$ & $80,1 \pm 4,1$ & $0,30 \pm 0,53$ & 0,129 & $79,9 \pm 3,3$ & $79,6 \pm 3,3$ & $-0,25 \pm 0,33$ & $<0,05$ & $0,55 \pm 0,50$ & 0,05 \\
\hline $\begin{array}{l}\text { Suma de } \\
6 \text { pliegues } \\
(\mathrm{mm})\end{array}$ & $51,3 \pm 12,2$ & $48,2 \pm 16,7$ & $-3,1 \pm 14,0$ & 0,521 & $108,3 \pm 32,0$ & $79,9 \pm 11,3$ & $-30,4 \pm 40,3$ & $<0,001$ & $27,3 \pm 25,4$ & $<0,001$ \\
\hline $\begin{array}{l}\text { Masa tejido } \\
\text { adiposo }(\mathrm{kg})\end{array}$ & $13,4 \pm 1,5$ & $13,3 \pm 2,1$ & $-0,10 \pm 2,7$ & 0,915 & $21,3 \pm 4,3$ & $17,1 \pm 2,5$ & $-4,3 \pm 5,4$ & $<0,001$ & $4,2 \pm 3,7$ & $<0,001$ \\
\hline $\begin{array}{l}\text { Masa } \\
\text { muscular (kg) }\end{array}$ & $26,8 \pm 2,6$ & $28,2 \pm 2,5$ & $1,42 \pm 1,4$ & $<0,05$ & $28,0 \pm 4,4$ & $29,6 \pm 3,4$ & $1,59 \pm 1,9$ & $<0,05$ & $0,17 \pm 0,23$ & 0,850 \\
\hline $\begin{array}{l}\text { Masa ósea } \\
(\mathrm{kg})\end{array}$ & $5,8 \pm 0,65$ & $6,0 \pm 0,6$ & $0,22 \pm 0,42$ & 0,162 & $6,1 \pm 0,89$ & $5,9 \pm 0,83$ & $-0,24 \pm 0,19$ & $<0,01$ & $0,46 \pm 0,50$ & $<0,05$ \\
\hline $\begin{array}{l}\text { Índice } \\
\text { músculo/ } \\
\text { óseo (kg) }\end{array}$ & $4,6 \pm 0,31$ & $4,71 \pm 0,34$ & $0,07 \pm 0,36$ & 0,596 & $4,6 \pm 0,28$ & $5,1 \pm 0,46$ & $0,49 \pm 0,44$ & $<0,01$ & $0,42 \pm 0,39$ & 0,144 \\
\hline $\begin{array}{l}\text { Masa adiposa } \\
(\%)\end{array}$ & $24,5 \pm 0,17$ & $23,5 \pm 0,32$ & $-0,1 \pm 0,41$ & 0,440 & $33,1 \pm 0,61$ & $27,4 \pm 0,16$ & $-5,7 \pm 7,1$ & $<0,001$ & $5,6 \pm 3,42$ & $<0,001$ \\
\hline $\begin{array}{l}\text { Masa } \\
\text { muscular (\%) }\end{array}$ & $48,9 \pm 0,13$ & $49,8 \pm 0,32$ & $0,92 \pm 2,9$ & 0,365 & $43,2 \pm 0,41$ & $47,8 \pm 0,19$ & $4,6 \pm 5,41$ & $<0,001$ & $3,68 \pm 2,36$ & $<0,001$ \\
\hline $\begin{array}{l}\text { Masa ósea } \\
(\%)\end{array}$ & $10,0 \pm 0,01$ & $10,1 \pm 0,01$ & $0,1 \pm 0,71$ & 0,856 & $9,32 \pm 0,18$ & $10,06 \pm 0,72$ & $0,74 \pm 0,75$ & $<0,05$ & $0,89 \pm 0,70$ & 0,053 \\
\hline Endomorfía & $2,4 \pm 0,73$ & $2,1 \pm 1,0$ & $-0,26 \pm 0,56$ & 0,197 & $4,7 \pm 1,2$ & $3,5 \pm 0,84$ & $-1,2 \pm 1,7$ & 0,060 & $0,94 \pm 1,01$ & 0,094 \\
\hline Mesomorfía & $3,0 \pm 0,81$ & $3,1 \pm 0,74$ & $0,08 \pm 0,15$ & 0,137 & $4,2 \pm 0,58$ & $4,3 \pm 0,51$ & $0,06 \pm 0,33$ & 0,561 & $0,02 \pm 0,01$ & 0,890 \\
\hline Ectomorfía & $3,2 \pm 1,0$ & $2,9 \pm 0,98$ & $-0,28 \pm 0,73$ & 0,285 & $1,4 \pm 0,47$ & $1,9 \pm 0,63$ & $0,44 \pm 0,66$ & 0,079 & $0,72 \pm 0,52$ & $<0,05$ \\
\hline SJ $(\mathrm{cm})$ & $31,9 \pm 3,6$ & $31,8 \pm 3,3$ & $-0,12 \pm 3,3$ & 0,914 & $27,6 \pm 2,5$ & $28,4 \pm 3,7$ & $0,77 \pm 3,6$ & $<0,001$ & $0,89 \pm 0,54$ & 0,634 \\
\hline $\mathrm{CMJ}(\mathrm{cm})$ & $37,4 \pm 5,5$ & $37,9 \pm 4,8$ & $0,48 \pm 3,7$ & 0,712 & $30,5 \pm 2,7$ & $31,4 \pm 3,2$ & $0,92 \pm 2,9$ & 0,380 & $0,44 \pm 0,39$ & 0,783 \\
\hline ABLKJ $(\mathrm{cm})$ & $42,5 \pm 5,2$ & $43,5 \pm 5,4$ & $1,0 \pm 1,7$ & 0,105 & $35,6 \pm 3,2$ & $36,1 \pm 4,9$ & $0,53 \pm 3,6$ & $<0,001$ & $0,47 \pm 0,24$ & 0,647 \\
\hline $\mathrm{MJ}(\mathrm{cm})$ & $45,4 \pm 4,9$ & $48,6 \pm 4,6$ & $3,1 \pm 2,9$ & $<0,05$ & $38,0 \pm 4,8$ & $41,5 \pm 5,4$ & $3,5 \pm 6,5$ & $<0,001$ & $0,40 \pm 0,38$ & 0,897 \\
\hline
\end{tabular}

GC, grupo control; GE, grupo experimental; BMI, índice de masa corporal; SJ, squat jump; CMJ, counter movement jump; ABLKJ, Abalakob jump; MJ, maximal jump. 
La CS, aunque mejoró en $75 \%$ de los saltos en el grupo experimental (SJ, ABLKJ y MJ) y solo en el MJ para el grupo control, no muestra ser efecto de la restricción calórica (Tabla 4); posiblemente por una elevada dispersión de datos y una muestra, aunque representativa de la élite, fue muy pequeña. Además, la presencia de una base de entrenamiento y una experiencia importantes en esta muestra, parecen justificar los cambios pequeños en la CS. Siempre los grandes cambios tienden a ser observados en las primeras semanas de entrenamiento o en poblaciones menos entrenadas ${ }^{(2)}$.

Los análisis de correlación (Tabla 5) entre la CS y las variables que mostraron los mayores efectos por la restricción calórica fueron bastante potentes para los cuatro tipos de salto. Además, las correlaciones negativas observadas entre los tipos de salto, con la MC, IMC, $\Sigma 6$ skf, y MA (en kg y \%), indicarían que con un aumento de cualquiera de estos factores las jugadoras de fútbol saltan menos (Tabla 5). Esto concuerda con otros estudios, como el de Lesinski et al..$^{(2)}$ y Hazir ${ }^{(31)}$. En los cuales se describen también, las características antropométricas más favorables para el fútbol.

La pérdida de capacidad de salto con una mayor MC, IMC y MA (en $\mathrm{kg}$ y \%) es similar a lo observado en otros deportes ${ }^{(3,26,27,32)}$. Al comparar las características antropométricas con la CS en estudios con jugadores de fútbol masculino se observó una relación significativa entre la masa corporal con la MA en el tronco y la MA (\%) con la CS ${ }^{(27,32) .}$
En Colombia, es frecuente encontrar jugadoras de excelentes condiciones técnicas y talento para el fútbol, pero con sobrepeso o elevada cantidad de masa adiposa, por lo que estos resultados representan una realidad poblacional.

Al comparar el MJ con variables asociadas a la composición corporal, se mostró una mayor correlación $(\mathrm{r}$ $=-0,716 \Sigma 6 \mathrm{skf} ; r=-0,652 \mathrm{MA}(\mathrm{kg}) ; r=0,481 \mathrm{MM}$ (\%)) que con los otros tipos de salto. El MJ es el único salto que simula uno de los gestos deportivos del fútbol. Además, el uso de los brazos y los pasos de impulso previos, lo hacen más fácil de realizar por las jugadoras, sin verse muy afectadas por una mayor BM. Según los resultados se entiende que no es necesario tener una mayor MM para alcanzar mayor CS y, que la condición ectomorfa, tiene una elevada correlación con la CS. Por lo anterior se concluye que, mientras más altas y delgadas son las jugadoras, alcanzan una mayor JC (Tabla 5).

En conclusión, a menor masa adiposa mayor capacidad de salto; y mientras más altas y delgadas sean las jugadoras saltarán más, pero no parece ser requerida una mayor masa muscular para tener una mayor capacidad de salto. El entrenamiento unido a una restricción calórica leve muestra un potente efecto sobre la masa corporal, el índice de masa corporal y la masa adiposa expresada como suma de pliegues y $\mathrm{kg}$ y $\%$ jugadoras de fútbol con exceso de tejido adiposo. Sin embargo, no se encontró ningún efecto de la dieta sobre la capacidad de salto en estas jugadoras.

Tabla 5. Análisis de correlación entre la capacidad de salto (JC) y las diferentes variables antropométricas estudiadas. Se muestra el valor de correlación $(r)$ y el valor de significación $(p)$. Las diferencias significativas se señalan a partir de un valor de $p<0,05$

\begin{tabular}{|l|l|c|c|c|c|c|c|c|c|c|c|}
\hline \multicolumn{1}{|c|}{ Salto } & & MC & IMC & I6skf & MA (kg) & MA (\%) & MM (kg) & MM (\%) & ENDO & MESO & ECTO \\
\hline \multirow{2}{*}{ SJ } & $r=$ & $-0,565$ & $-0,674$ & $-0,612$ & $-0,575$ & $-0,439$ & $-0,370$ & - & $-0,573$ & $-0,701$ & 0,662 \\
\cline { 2 - 12 } & $p=$ & $<0,001$ & $<0,001$ & $<0,001$ & $<0,001$ & $<0,01$ & $<0,05$ & $\mathrm{n} / \mathrm{s}$ & $<0,001$ & $<0,001$ & $<0,001$ \\
\hline \multirow{2}{*}{ CMJ } & $r=$ & $-0,516$ & $-0,736$ & $-0,732$ & $-0,645$ & $-0,570$ & - & 0,384 & $-0,679$ & $-0,781$ & 0,788 \\
\cline { 2 - 12 } & $p=$ & $<0,01$ & $<0,001$ & $<0,001$ & $<0,001$ & $<0,001$ & $\mathrm{n} / \mathrm{s}$ & $<0,05$ & $<0,001$ & $<0,001$ & $<0,001$ \\
\hline \multirow{2}{*}{ ABLKJ } & $r=$ & $-0,521$ & $-0,718$ & $-0,707$ & $-0,632$ & $-0,583$ & - & 0,388 & $-0,671$ & $-0,712$ & 0,751 \\
\cline { 2 - 11 } & $p=$ & $<0,01$ & $<0,001$ & $<0,001$ & $<0,001$ & $<0,001$ & $\mathrm{n} / \mathrm{s}$ & $<0,05$ & $<0,001$ & $<0,001$ & $<0,001$ \\
\hline \multirow{2}{*}{ MJ } & $r=$ & $-0,448$ & $-0,605$ & $-0,716$ & $-0,652$ & $-0,630$ & - & 0,481 & $-0,620$ & $-0,577$ & 0,625 \\
\cline { 2 - 11 } & $p=$ & $<0,01$ & $<0,001$ & $<0,001$ & $<0,001$ & $<0,001$ & $\mathrm{n} / \mathrm{s}$ & $<0,01$ & $<0,001$ & $<0,001$ & $<0,001$ \\
\hline
\end{tabular}

MC, masa corporal; $\Sigma 6 \mathrm{skf}$, suma de seis pliegues; IMC, índice de masa corporal; ENDO, endomorfa; MESO, mesomorfo; ECTO, ectomorfa. 
Este estudio hecho por primera vez en Colombia plantea otras posibilidades de investigación en la misma área y grupo deportivo, orientadas a mejorar el rendimiento de nuestras jugadoras, teniendo en cuenta el auge del futbol femenino en Colombia y en el mundo.

\section{APLICACIONES PRÁCTICAS}

El manejo nutricional de las jugadoras de fútbol profesional es importante para promover un mayor rendimiento físico-deportivo. Por ello, las jugadoras deben recibir educación nutricional desde el inicio de la práctica deportiva, que permita ajustar la dieta a sus necesidades energéticas, cargas de entrenamiento y principales necesidades nutricionales de la mujer atleta (calcio, hierro, ácido fólico, zinc). La educación nutricional permite ajustar la dieta manteniendo un bajo valor de tejido adiposo y un adecuado nivel de MM, que terminará beneficiando el propio rendimiento físico de las jugadoras. En caso de encontrar sobrepeso o exceso de tejido adiposo entre las jugadoras, se sugiere hacer una restricción calórica leve en su dieta (20\% a $25 \%$ menor al aporte energético recomendado), que ayudará a ajustar la composición corporal sin afectar el rendimiento físico. Por último, en la selección de talentos se debe tener en cuenta que las jugadoras longilíneas, con adecuado tejido adiposo (una $\Sigma 6 \mathrm{Skf}$ entre $40-60 \mathrm{~mm}$ ), podrán beneficiarse más del desarrollo de sus capacidades físicas, como el salto, la potencia y la velocidad de reacción, entre otras.

\section{Agradecimientos}

Queremos agradecer al equipo técnico, colaboradores y, en especial, a las jugadoras de la Selección Nacional de Colombia.

\section{Financiación}

El presente artículo no tuvo financiación externa de ningún tipo. Todas las actividades e insumos necesarios para la investigación se hicieron dentro de las tareas de trabajo diario con el Seleccionado Nacional.

\section{Conflicto de intereses}

Los autores declaran no tener conflicto de intereses.

\section{Declaración de autoría}

García-Morales G.I; Niño-Rey M.A; Alvear-Ordenes I. participaron en este estudio, todos los autores revisaron el artículo y validaron su versión final.

\section{Referencias bibliográficas}

1. Almagià FAA, Rodríguez RF, Barraza GFO, Lizana APJ, Jorquera ACA. Perfil antropométrico de jugadoras chilenas de fútbol femenino. Int J Morphol. 2008;26(4): 817-21.

2. Lesinski M, Prieske O, Helm N, Granacher U. Effects of soccer training on anthropometry, body composition, and physical fitness during a soccer season in female elite young athletes: a prospective cohort study. Front Physiol. 2017;8:1093.

3. Holway F. Composición corporal en nutrición deportiva. En: Boullosa MB, Peniche ZC. Nutrición Aplicada al deporte. Primera edición. España: McGraw Hill; 2011. p. 195-218.

4. Can F, Yilmaz I, Erden Z. Morphological characteristics and performance variables of women soccer players. J Strength Cond Res. 2004;18(3):480-5.

5. Reilly T, Bangsbo J, Franks A. Anthropometric and physiological predispositions for elite soccer. J Sports Sci. 2000; 18(9): 669-83.

6. Kreider RB, Wilborn CD, Taylor L, Campbell B, Almada AL, Collins $\mathrm{R}$, et al. ISSN exercise and sport nutrition review: research and recommendations. J Int Soc Sports Nutr. 2010;7:7.

7. Manson SA, Brughelli M, Harris NK. Physiological characteristics of international female soccer players. J Strength Cond Res. 2014; 28(2): 308-18.

8. Grupo de Trabajo sobre Nutrición del Comité Olímpico Internacional. Nutrición para deportistas información médica para deportistas: guía práctica para comer y beber, para mejorar la salud y el rendimiento físico. Conferencia Internacional de Consenso COI, Lausana 2012. (Consultado el 1 de agosto 2019). Disponible en : http://deporte.aragon.es/recursos/ files/documentos/doc-areas_sociales/deporte_y_salud/ guia_nutricion_deportistas.pdf

9. Maughan R, Burke L, Kirkendall DT. F-MARC. Nutrición para el futbol. Conferencia Internacional de consenso. FIFA. Zurich. 2005; 4-17; 32-33. (Consultado el 1 de agosto 2019) Disponible en : https://es.scribd.com/document/397400727/MANUAL-DE-MEDICINA-DELFUTBOL-PDF-

10. Grimm K, Kirkendall DT. Health and fitness for the female football player. Football food - promoting health, fitness and performance. FIFA Medical Assessment and Research Centre (F-MARC) 2007. 45-55. (Consultado el 1 de Agosto 2019) Disponible en: https://resources.fifa.com/image/upload/ female-player-booklet-1452572.pdf?cloudid=thc79bbqdf5g 6qnpcaib.

11. Thomas DT, Erdman KA, Burke LM. Position of the Academy of Nutrition and Dietetics, Dietitians of Canada, and the American College of Sports Medicine: Nutrition and Athletic Performance. J Acad Nutr Diet. 2016;116(3):501-28.

12. Coker RH, Miller S, Schutzler S, Deutz N, Wolfe RR. Whey protein and essential amino acids promote the reduction of adipose tissue and increased muscle protein synthesis during 
caloric restriction-induced weight loss in elderly, obese individuals. Nutr J. 2012; 11:105.

13. Bosco C, Luhtanen P, Komi PV. A simple method for measurement of mechanical power in jumping. Eur J Appl Physiol Occup Physiol. 1983;50(2):273-82.

14. Oddsson L, Thorstensson A. Evaluation of physical performance. En: Ekblon Bj. Football (Soccer). Handbook of Sports Medicine and Science's series. Primera Edición. Oxford:Wiley-Blackwell, 1994. p. 102-120.

15. StewartA,Marfell-Jones M,Olds T,DeRidderJH.International Standards for Antropometric Assessment. International Sciety for the Advancement of Kinanthropometry: New Zealand; 2011.

16. Ross WD, Kerr DA. Fraccionamiento de la masa corporal: Un nuevo método para utilizar en nutrición clínica y medicina deportiva. Apunts Med Esport. 1991;28:175-87.

17. Centeno-Prada RA, López C, Naranjo-Orellana J. Jump percentile: a proposal for evaluation of high-level sportsmen. J Sports Med Phys Fitness. 2015; 55(5):464-70.

18. Rodriguez NR, DiMarco NM, Langley S, American Dietetic Association, Dietitians of Canada, American College of Sports Medicine: Nutrition and Athletic Performance. Position of the American Dietetic Association, Dietitians of Canada, and the American College of Sports Medicine: Nutrition and athletic performance. J Am Diet Assoc. 2009; 109(3):509-27.

19. Roza AM, Shizgal HM. The Harris Benedict equation reevaluated: resting energy requirements and the body cell mass. Am J Clin Nutr. 1984; 40(1):168-82.

20. Ainsworth BE, Haskell WL, Whitt MC, Irwin ML, Swartz AM, Strath SJ, et al. Compendium of physical activities: an update of activity codes and MET intensities. Med Sci Sports Exerc. 2000; 32(9 Suppl):S498-504.

21. FIFA. Copa mundial de la FIFA [Internet]. Zurich: 2017. [citado 10 Nov 2017] Disponible en: http://es.fifa.com/fifatournaments/statistics-and-records/worldcup/index.html

22. Sedano S, Vaeyens R, Redondo JC. The Relative Age Effect in Spanish Female Soccer Players. Influence of the Competitive Level and a Playing Position. J Hum Kinet. 2015; 46:129-37.

23. Martin $\mathrm{AD}$. An anatomical basis for assessing human body composition: evidence from 25 dissections [Internet]. [PhD
Thesis]. Simon Fraser University, Canada. 1984. (Consultado el 1 de Agosto 2019). Disponible en: https://core.ac.uk/ download/pdf/56370706.pdf

24. Mojtahedi MC, Thorpe MP, Karampinos DC, Johnson CL, Layman DK, Georgiadis JG, et al. The effects of a higher protein intake during energy restriction on changes in body composition and physical function in older women. J Gerontol A Biol Sci Med Sci. 2011; 66(11):1218-25.

25. Mahan LK, Raymond JL. Krause's food \& the nutrition care process. Fourteenth edition. St. Louis, Missouri: Elsevier, 2017.

26. Blumenthal JA, Babyak MA, Sherwood A, Craighead L, Lin $\mathrm{PH}$, Johnson J, et al. Effects of the dietary approaches to stop hypertension diet alone and in combination with exercise and caloric restriction on insulin sensitivity and lipids. Hypertension. 2010;55(5):1199-205.

27. Orquín CFJ, Torres-Luque G, Ponce de León F. Efectos de un programa de entrenamiento de fuerza sobre la composición corporal y la fuerza máxima en jóvenes entrenados. Apunts Med Esport. 2009; 44(164):156-62.

28. Kreider RB, Serra M, Beavers KM, Moreillon J, Kresta JY, Byrd $\mathrm{M}$, et al. A structured diet and exercise program promote favorable changes in weight loss, body composition, and weight maintenance. J Am Diet Assoc. 2011;111(6):828-43.

29. Milanovic Z, Sporis G, Trajkovic N, James N, Samija K. Effects of a 12-week SAQ Training Program on agility with and without the ball among young soccer players. J Sports Sci Med. 2013;12(1):97-103.

30. Milanovic Z, Sporis G, Trajkovic N. Differences in body composite and physical match performance in female soccer players according to team position. J Hum Sport Exerc. 2012;7 (Proc1):S67-S72.

31. Hazir T. Physical characteristics and somatotype of soccer players according to playing level and position. J Hum Kinet. 2010; 26: 83-95.

32. Garrido CRP, González LM, García VM, Expósito CI. Correlación entre los componentes del somatotipo y la composición corporal según fórmulas antropométricas [Internet]. 2005 [citado 4 Feb 2017]. Disponible en: www.efdeportes. com/efd84/somato.htm 